\title{
CURRÍcuLo PARA LA FORMACIÓN EN INVESTIGACIÓN, INNOVACIÓN Y PENSAMIENTO SOCIO-CRÍTICO APOYADO EN TIC
}

\section{CURRICULUM FOR'TRAINING RESEARCH, INNOVATION AND SOCIO-CRI'TICAL'THINKING SUPPOR'TES BY'TIC}

\author{
Mawency Vergel Ortega ${ }^{1}$ \\ Jhan PieroRojas Suarez ${ }^{2}$ \\ Victor Jhoel Bustos Urbano ${ }^{3}$
}

\section{RESUMEN}

La investigación parte de un análisis documental con enfoque cualitativo. Su objetivo es diseñar la tendencia curricular para orientar cursos, módulos y asignaturas de los diferentes programas académicos de la universidad Francisco de Paula Santander ubicada en Cúcuta Colombia. La tendencia curricular validada se centra en el desarrollo de competencias y habilidades desde el enfoque dialógico crítico orientado a la formación en investigación e

\footnotetext{
$1 \quad$ Postdoctora en Ciencias sociales, niñez y juventud, Doctora en educación. Filiación: Universidad Francisco De Paula Santander, Cúcuta, Colombia. Correo: mawencyvergel@ufps.edu.co https://orcid.org/0000-0001-8285-2968

$2 \quad$ Magister en Esructuras. Filiación: Universidad Francisco De Paula Santander Cúcuta, Colombia correo: jhanpierorojas@ufps.edu.co https://orcid.org/0000-0003-2682-9880

3 Magister en Gobierno y gestión pública. Filiación: Universidad Francisco De Paula Santander Cúcuta, Colombia. correo: Victorjhoelbu@ufps.edu.co https://orcid.org/0000-0001-6954-2260
} 
innovación apoyado en las tecnologías de la comunicación. En conclusion, la tendencia ha fomentado en estudiantes y profesores el desarrollo de habilidades de pensamiento formal, crítico, creatividad, generación de ideas para el emprendimiento, desarrollo de investigaciones desde tic, diseño y generación de prototipos para la innovación.

PALABRAS CLAVE: tendencia curricular, innovación, transformación social, universidad, tecnologias

\section{ABSTRACT}

The research is based on a documentary analysis, a qualitative approach. Its objective is to design the curricular trend to guide courses, modules and subjects of the different academic programs of the Francisco de Paula Santander University located in Cúcuta Colombia. The validated curricular trend is focused on the development of competences and skills from the critical dialogic approach oriented to research and innovation training supported by communication technologies. Conclusion: the tendency has promoted in students and teachers the development of skills of formal thought, creativity, generation of ideas for the enterprise, development of investigations from tic, design and generation of prototypes for the innovation.

KEYWORDS: curricular trend, innovation, social transformation, university, technologies

\section{INTRODUCCIÓN}

El currículo en el marco de enfoques y modelos pedagógicos inspirados en las pedagogías críticas, ha centrado su apuesta en la formación de sujetos innovadores, sociales y políticos a partir de una perspectiva sociocrítica de la sociedad y la educación (interés emancipatorio). Etimológicamente se entiende por curriculum "una carrera o curso", entendido como "course of subject matter studies". El concepto "curriculum vitae" no es sino la "carrera de vida" Con Col entendemos por curriculum "el proyecto que preside las actividades educativas escolares, precisa sus intensiones y proporciona guías de acción adecuadas y útiles para los profesores que tiene la responsabilidad directa de su ejecución". Por su parte Gimeno [2], afirma que el "curriculum es el eslabón entre la cultura y la sociedad exterior a la escuela y la educación, entre el conocimiento o la cultura heredados y el aprendizaje de los alumnos, entre la teoría (ideas, supuestos y aspiraciones) y práctica posible, dadas unas determinadas condiciones". Y puntualiza aún más: "El curriculum es la expresión y concreción del plan cultural que una institución escolar hace realidad dentro de unas determinadas condiciones que matizan ese proyecto".

Para la investigación se tomaron experiencias de programas académicos en la Universidad Francisco de Paula Santander. Los materiales analizados fueron entrevistas en profundidad, semiestructuradas, notas de observación participante, y análisis de fuentes documentales, físicas y virtuales. En torno al conjunto de criterios, se consolidan programas académicos y sus correspondientes planes de estudio, programas, metodologías, y procesos que contribuyen a la formación de calidad y a la construcción de escenarios que propician la identidad cultural nacional, regional y local, en el cual, la institución, se apoya de actores y recursos académicos y físicos, para poner en práctica las políticas educativas del Ministerio de Educación Nacional, así como políticas en el ámbito internacional que permiten la movilidad de los actores del proceso educativo para dar cumplimiento al proyecto educativo institucional y a la misión y visión de la Universidad Francisco de Paula Santander.

En torno a ello, en el presente documento se registran ideas que orientan en la dirección referida, a las concepciones de educación que han estado presentes en quehacer y en 
programas académicos, donde el currículo es el elemento mediador entre la teoría educativa y su práctica, para dar solución a problemas sociales y promover el desarrollo económico de un país, en particular de la región de frontera que atiende ademas a la población del Catatumbo, de manera que, la institución y su programas ofertados muestran una constante innovación, se exponen entonces algunas de las tendencias en materia curricular y la medida en que éstas se han venido aplicando independientemente de las fuentes de origen; tendencias curriculares presentes en los programas académicos que la región demanda, como escenarios y retos que se anteponen en diferentes modelos y formas de construcción del conocimiento y los entornos que lo generan.

\section{METODOLOGIA}

una perspectiva que en la visión de Hernández [3], y Vergel et.al [4] analiza documentos y prácticas en contextos virtuales, y a distancia, sincrónicos y asincrónicos; utiliza la compilación de información para probar hipótesis, con base en la medición numérica y el análisis estadístico, para establecer estándares de comportamiento y probar teorías. En este orden de ideas, la investigación fue primero documental, exploratoria, luego descriptiva [5] y correlativa. Además, la investigación se realizó en cuatro fases: valoración y diagnóstico de la situación actual, revisión de la literatura para construir la condición del arte, diseño y desarrollo de la tendencia, para llegar finalmente a la evaluación de los impactos en estas cuatro fases. El artículo se centra en la fase uno de análisis de precedentes, análisis cualitativo, de generación de categorías que dan surgimiento a la tendencia y sus interacciones, la búsqueda de la condición del arte y la descripción de la condición actual de la problemática [6], en esta etapa se establecerá una caracterización de la población beneficiaria. Una segunda fase de la investigación consiste en el diseño de la técnica, desde la misión, la visión, los valores, el inicio y las políticas y la planificación estratégica para la funcionalidad en condiciones de emergencia declarada por COVID 2019. Asimismo, en esta fase se diseñan mapas de proceso para el área asociada a matemáticas, se analizan currículos y objetivos, perfiles profesionales. Y propósito institucional acorde además al desarrollo evidente de procesos inmersos en investigación e innovación. En la fase de implementación y funcionamiento se visualiza avances en implementación de la tendencia, mediante estrategias y caracterizando los estudiantes como base piloto a partir de datos en riesgo matemático. Se inicia la implementación de técnicas y herramientas de enseñanza, aprendizaje, así como de evaluación y atención, durante el proceso de aprendizaje desde escenarios sincrónicos y asincrónicos. Finalmente, la fase de valoración del impacto futuro, a fin de prevé acciones correctivas que mejoren todo aquello que subyace a la tendencia curricular.

\section{RESULTADO Y DISCUSIÓN}

\section{Concepciones de Currículo asociados a la} tendencia. Zabalza [7] considera que el término currículo puede entenderse desde tres posibles enfoques: a. El currículo como normativa sobre la estructuración de los estudios a realizar por los alumnos en los diferentes niveles de enseñanza. Su eje estructural es la planificación a nivel de sistema educativo y abarca aspectos como la planificación educativa, la organización escolar, el diseño y evaluación de programas. b. El currículo como conjunto de oportunidades de aprendizaje ofertados a la juventud del Catatumbo en situaciones concretas [8]. Explica lo que se pretende lograr en una situación de enseñanza aprendizaje, para alcanzar los propósitos formativos. Incluye aspectos concretos tales como: la determinación de los objetivos de aprendizaje, la selección y organización de los contenidos, la previsión 
de las actividades a realizar o experiencias a ofertar a los alumnos, el diseño y manejo de materiales didácticos, la evaluación de los resultados. Se trata de un concepto de currículo basado en la programación. c. El currículo como proceso educativo real, que se desarrolla en un contexto particular de enseñanza (centro, aula). Su pretensión es superar las desconexiones entre lo programado y lo que realmente se hace[9]. Existe según ello un currículo formal (previsiones) y un currículo real (realizaciones). La cuestión básica radica, no en lo que es el currículo formal, sino en cómo se usa. Sus aspectos básicos son: contexto del desarrollo curricular, dinámica ideográfica del aula, ecología curricular, principios de procedimiento.

Clasificación del concepto currículo En la UFPS el término currículo a nivel institucional, es analizado desde tres perspectivas: $a$. Un ámbito configurado como conjunto de finalidades, secuencias de contenidos y actividades que orientan la tarea del profesor en el aula y en el centro: el estudiante. Estaría determinado por tendencias pedagógicas y los programas autoevaluados continuamente interrelacionados con currículos del ámbito internacional, entendidos como planificación de la enseñanza, a nivel autonómico. b. Un segundo nivel, está caracterizado por los proyectos curriculares [10]. Los cuales buscan orientar la identidad pedagógica - didáctica soportada en un modelo dialógico-crítico. Un tercer nivel se concreta en el diseño curricular de aula a programación de cada profesor y equipo docente, como una concreción de las orientaciones pedagógicas y didácticas generales. Esta triple estructura es la que prevalece en los diseños curriculares.

La concepción de currículo se asocia además a espacio público a partir de la relación investigación gestión, en ella el currículo es de todos y para todos y es construido por todos, es base para su construcción, la investigación. Por lo tanto, el currículo es concebido como un proceso de innovación, investigación, creación y desarrollo continuo, como un acontecer que es dinámico, participativo, crítico, creativo e investigativo, que se materializa cuando el agente educativo se enfrenta a su ámbito institucional y desarrolla actividades significativas para él y para la sociedad en su conjunto. El currículo es el marco integrador de las funciones universitarias que logra su concreción en escenarios de aprendizaje derivados de procesos de construcción colectiva, que se realizan en función del saber-hacer disciplinario, interdisciplinario, multidisciplinario y transdisciplinario integrados y relacionados a currículos en el ámbito nacional e internacional en el marco de las políticas educativas establecidas por el Ministerio de Educación Nacional, por el Ministerio de las Tic y del Ministerio de Ciencia, tecnología e innovación (Colciencias). Para lograr esto, el currículo debe ser comprendido como una edificación permanente de sabereshaceres y quehaceres que tienden un puente estructurador y ordenativo entre la teoría y la práctica desde una visión contextualizada en sus dimensiones histórica, sociocultural y política.

Lacreación delos diseñosyrediseños curriculares se sustentan en dos vertientes: la innovación e investigación como estamento fundamental de las actividades y procesos de construcción, y bajo el enfoque de diseños curriculares por competencias. La investigación como base del currículo la convierte en una función esencial en lo relativo al avance del conocimiento, a su aplicación y para la búsqueda de soluciones a problemas específicos. Se concibe como una actividad integrada y en interacción permanente con las actividades de docencia, extensión y proyección social en el marco de procesos de inclusión y del establecimiento de políticas que conllevan al desarrollo de la región y al fomento de la innovación y de la paz.

El currículo así concebido permite flexibilizar y autorregular los planes de estudio, y la 
movilización de los diferentes procesos de interacción cognoscitiva y programática al impulsar dinámicas de empoderamiento presentes en el currículo. En este sentido, este proceso de transformación curricular reconoce la investigación como actividad productiva que permite repensar técnicas educativas y modelos educativos de los programas académicos, lo que significa que el modelo pedagógico dialógico crítico y sus lineamientos curriculares se centra en el saber-hacer innovativo-investigativo pertinente y resolutivo dentro de una formación que propicia el diálogo de saberes y el pensamiento crítico de los estudiantes. En este sentido, la actividad investigativa construye opciones articuladas a diversos escenarios de trabajo y estudio en auténticos diálogos entre saberes. En consecuencia, procesos formativos, de extensión y la gestión, en tanto procesos naturales de la actividad humana, deben derivar en prácticas académicas para el desarrollo de competencias y habilidades impregnadas de contenido creativo e investigativo para impulsar transposiciones didácticas del saber académico desde la formación básica hasta la profesional, en el contexto del conocimiento acorde a perfiles profesionales de egreso por competencia y habilidad. Desde aquí se infiere la necesidad de implementar un modelo curricular problematizador como proceso de trabajo investigativo, basado en la búsqueda permanente de respuestas ante los hechos educativos, profesionales y sociales. De otra parte, el enfoque profesional curricular de innovación por competencias de los programas se adhiere a las tendencias internacionales, las cuales indican que dicho enfoque es una opción efectiva para la formación.

Concepciones de competencia hacia el emprendimiento y la formación en el agro, la ciencia, la ingeniería o la administración, pasan a ser, la estructura dinámica organizadora de la actividad y son asumidas desde como procedimientos complejos que reflejan las demostraciones inherentes a las condiciones personales y profesionales que desarrollan en las diferentes áreas de formación con calidad y pertinencia a través de un currículo que se sustenta en la actividad investigativa, de proyección social hacia la innovación y generación de nuevo conocimiento, con el fin de justificar y garantizar una práctica pedagógica articulada de forma integral las dimensiones académicas y sociales del currículo con el mundo real, para garantizar su pertinencia, calidad, dinámica, flexibilidad y la mejora continua.

\section{DIMENSIÓN ONTOLÓGICA}

En el análisis de tendencias curriculares presentes en los programas ofertados por la Universidad Francisco de Paula Santander, la dimensión ontológica incorpora el conocimiento y la aplicabilidad del campo de la ciencia y formación básica y profesional para el desarrollo de competencias y habilidades cuya evolución ha generado una trascendencia social con una dimensión muy dinámica en el campo de la ciencia. Desde que el hombre comienza su interrelación y dinámica por satisfacer sus necesidades data la transferencia de un conocimiento, con base científica y postulados axiomáticos, producto de una reflexión epistémica in situ de lo que ocurre en su realidad e hipótesis puestas en prueba para verificar soluciones a problemáticas presentes en la región en torno a la solución del conflicto y la búsqueda de escenarios de desarrollo en medio de la implementación de proyectos para la generación y construcción de paz. El realismo social que se vive diariamente desde el punto de vista que ofrece la nueva episteme indica la existencia de realidades múltiples y socialmente construidas. Este planteamiento se corresponde con lo expresado por Balza (2010), cuando enfatiza que "...la realidad se presenta indivisa y sin fronteras, siempre sujeta a lo que es nuestro pensamiento" (p. 23). Bajo esta perspectiva la naturaleza de la realidad del fenómeno de 
estudio es compleja, es decir, multidimensional y evolutiva. Tal como lo argumenta Morín (2002), citado por Balza (ob. cit.):

En la construcción del conocimiento acerca de la realidad, es preciso tener en cuenta que la realidad no es simple ni compleja. La realidad es lo que es nuestro pensamiento. Si nuestro pensamiento es simple, la realidad va a ser simple, si nuestro pensamiento es complejo, entonces la realidad va a ser compleja. (p. 35).

La realidad referente en el currículo de pregrado y su aplicabilidad social será el producto del desempeño de los estudiantes al utilizar los saberes en su devenir diario. En este contexto, la vida cotidiana se presenta como una realidad interpretada y para ello es necesario que el docente realice su praxis desde aspectos socio cualitativos y desde los enfoques cuantitativos para generar aprendizajes verdaderos y más objetivo de un mundo desarrollo de conocimiento, global, glocal, en contextos de innovación tecnológica - curricular y en el boom de una sociedad postmoderna donde el manejo de la tecología, la información, la investigación y la generación del conocimiento se han convertido en factores cruciales de la competitividad.

\section{Currículo para la formación en investigación e innovación y el pensamiento socio-crítico apoyado en TIC}

Se destaca, la constitución de un currículo investigativo para la formación de pensamiento socio-crítico, articulado con prácticas deliberativas en la investigación: la práctica pedagógica acompaña todos los espacios a partir de radicalizar el diálogo estudiantes - maestros en la formulación de investigaciones que propicien el desarrollo y producción de proyectos en torno a un conocimiento con sentido: grupos interdisciplinarios, asesoría interdisciplinar, colectivos, consolidación de semilleros. Acorde a postulados de Saldarriaga (2016), la articulación entre producción científica y tecnológica, apropiación de conocimiento y deliberación, entre currículo y democracia, se constituye en el eje de esta tendencia (Figura 1)

Esta tendencia implica en sus programas y correspondientes planes de estudio, crear e impulsar sentidos distintos de aprendizaje, ciencia y conocimiento, no funcionales al sistema hegemónico. Propone la producción participativa y deliberante de conocimiento para la solución de problemáticas en diferentes contextos para la emancipación social; no solo de reproducción, implica desarrollar mecanismos que movilicen el deseo de saber y el interés por lo emancipatorio, como dinamizadores de procesos de subjetivación. El reconocimiento de los estudiantes como sujeto de saber, en un proceso de subjetivación que apunta a la liberación de ese deseo motivado de saber (como heterotopía) y resolver problemáticas, convirtiéndoles en gestores de saber transformativo, favorece la ruptura con procesos de sujeción propios del mundo educativo de modelos pedagógicos de siglos pasados: pautar y pausar la experiencia, el deseo de conocer a los marcos y ritmos curriculares.

La incorporación de TIC en la tendencia configura la implementación de espacios educativos virtuales, semipresenciales, y presenciales, al permitir no solo el uso sino la creación de plataformas, software, aplicativos, juegos, blogs $y$ demás herramientas didácticas que se integran a las metodologías y tendencias 
educativas utilizadas por la comunidad académica. La comunicación digital, las teleconferencias, videos, videochats, la creación y uso masivo de la Plataforma PLAD, plataforma Uvirtual, de plataformas académicas como Coursera y colaborativas, la participación en redes cuya comunicación se potencia a través de la web, el uso de la nube para difusión y uso de material pedagógico-didácticocomunicación, de herramientas web, la producción de software, blogs, ha señalado un impacto en los procesos educativos, en el desarrollo del pensamiento creativo, en el rendimiento académico y en la satisfacción de jóvenes en formación como apoyo de la práctica pedagógica y a la asesoría académica, que se desarrolla en cada programa desde cada módulo o ara de conocimiento enseñado, lo anterior, de acuerdo a investigaciones realizadas. En la UFPS es aceptado por la comunidad la necesidad de implementar avances tecnológicos, sus implicaciones y el papel del ser humano, por ello la tecnología se ha convertido en un actor que participa de los procesos de formación, la sociedad y la cultura de la información y la virtualidad, siendo una herramienta primordial en la obtención de información que debe evaluarse y actualizarse continuamente. Por otra parte, el proceso educativo se confunde cada vez más con el trabajo.

Así mismo, la tendencia incorpora la generación de competencias comunicativas y de difusión de resultados de proyectos de investigación o de procesos de indagación, en una mirada más allá de conocimientos, de contenido sino de gestión del conocimiento, acorde a necesidades y perfiles de formación profesional en el cual toda la comunidad se integra para apoyar perspectivas que potencian y responden a una visión del ciudadano, acciones que gestionan sus habilidades, sus conocimientos, de manera que al egresar, pueden comunicar su saber a fin de superar obstáculos epistemológicos presentes en el aprendizaje de las diferentes áreas y ciencias y de aquellos que se encuentran entre el desarrollo tecnológico, el trabajo y el estudio.

Esta tendencia se apoya en tendencias educativas como el big data, wearable, inteligencia artificial, telepresencia, aprendizaje en la nube, videojuegos o retos desde la web. La idea según la cual es necesario acumular muchos y variados conocimientos va quedando atrás, lo importante es saber cómo aprenderlos en el momento en que se necesiten, saber dónde encontrarlos y cómo obtenerlos, pues el conocimiento por sí mismo no es el objetivo final, éste tiene sentido en la medida de su aplicación y vigencia. Este orden fortalece la relación del estudiante con su entorno, con sus dos universos: exterior e interior, lo que hace necesario reconocerse en el ámbito virtual y de uso de tic: los currículos interactivos, simuladores para la enseñanza, bibliotecas electrónicas, repositorios, redes de información, redes de revistas científicas, tesauros, y tecnología emergente; para los cuales la exigencia de nuevos entornos y rol docente, estudiante e institución, así como distintos lenguajes y competencias en la formación, son retos que se enfrentan y potencian continuamente.

Por otra parte, emergen sistemas asociados a la robótica y la presencia ausencia desde escenarios de virtualidad, telepresencia o realidad aumentada, los soportes multimedia y el análisis del lenguaje audiovisual e informatizado, específicamente centrados en la imagen y el audio. Resulta, entonces, imperioso su conocimiento, ya que la información se obtiene mediante lenguajes amigables y de una manera especial, a través del lenguaje visual. Ventajas 
de las tecnologías se centran, según Cabero, J. (2002), en: la simulación de fenómenos; la interactividad que permite adquirir un sentido pleno en el terreno educativo y didáctico; la instantaneidad; la digitalización; el desarrollo del proceso asociativo, la diversidad de información y de maneras de obtenerla.

Su apuesta es convertirse en programas socialmente incluyentes. En los procesos de formación propuestos, se da un giro particular por la evaluación académica: el paso intencionado de la heterorregulación a la autorregulación, disminuyendo el énfasis sobre los dispositivos de sanción y fortalece los de aprendizaje (satisfacción) mediante el autoexamen, como "un elemento de un sistema doble: gratificaciónsanción. $Y$ este es el sistema que se vuelve operante en el proceso de encausamiento de la conducta y de corrección" [12]. Esta apuesta se visualiza en la figura 1.

El currículo además incorpora proyectos, propuestas y escenarios alternativos para jóvenes con tendencia a la exclusión por razones de raza, ideología, género, nivel de desarrollo cognitivo o psicosocial, nivel económico, condición física o psicológica; excluidos del sistema educativo acorde a Saldarriaga [15], desplazados, víctimas de la violencia, víctimas de desastres naturales, no aceptados por la sociedad (comunidades afro, discapacidad, LGBTI, feminismo, autismo, hipoacusticos [16], jóvenes en embarazo, consumidores de psicoactivos, o expulsados de otras instituciones) [17].

Además, se construyen escenarios para transformar concepciones [18] y prácticas generadoras de violencias: asesorías jurídicas, colectivos de estudiantes mediadores de conflictos, autoevaluación de los estudiantes, así como la realización de encuentros sobre la diversidad sexual y de género, el bullying, las necesidades sociales, políticas, humanas, convocados por estudiantes. Esta tendencia muestra rasgos propios de los procesos de subjetivación, al horizontalizar la autoridad en ciertas decisiones de disciplina, e impulsa el reconocimiento de los sujetos considerados socialmente excluidos. Esta se enmarca en la dinámica cotidiana y sus reglas (ética, liderazgo, respeto, cumplimiento de compromisos), frente a una lógica de la libertad, la autoconciencia, el reconocimiento y valoración del otro [19], para vivir de experiencias, y aprender desde el interés propio a fin de potenciar habilidades de cogobierno y el cuidado de los otros [20]. Así, los estudiantes conforman grupos, semilleros, colectivos, asociaciones para la mediación y tramite de conflictos, con independencia de la autoridad. 


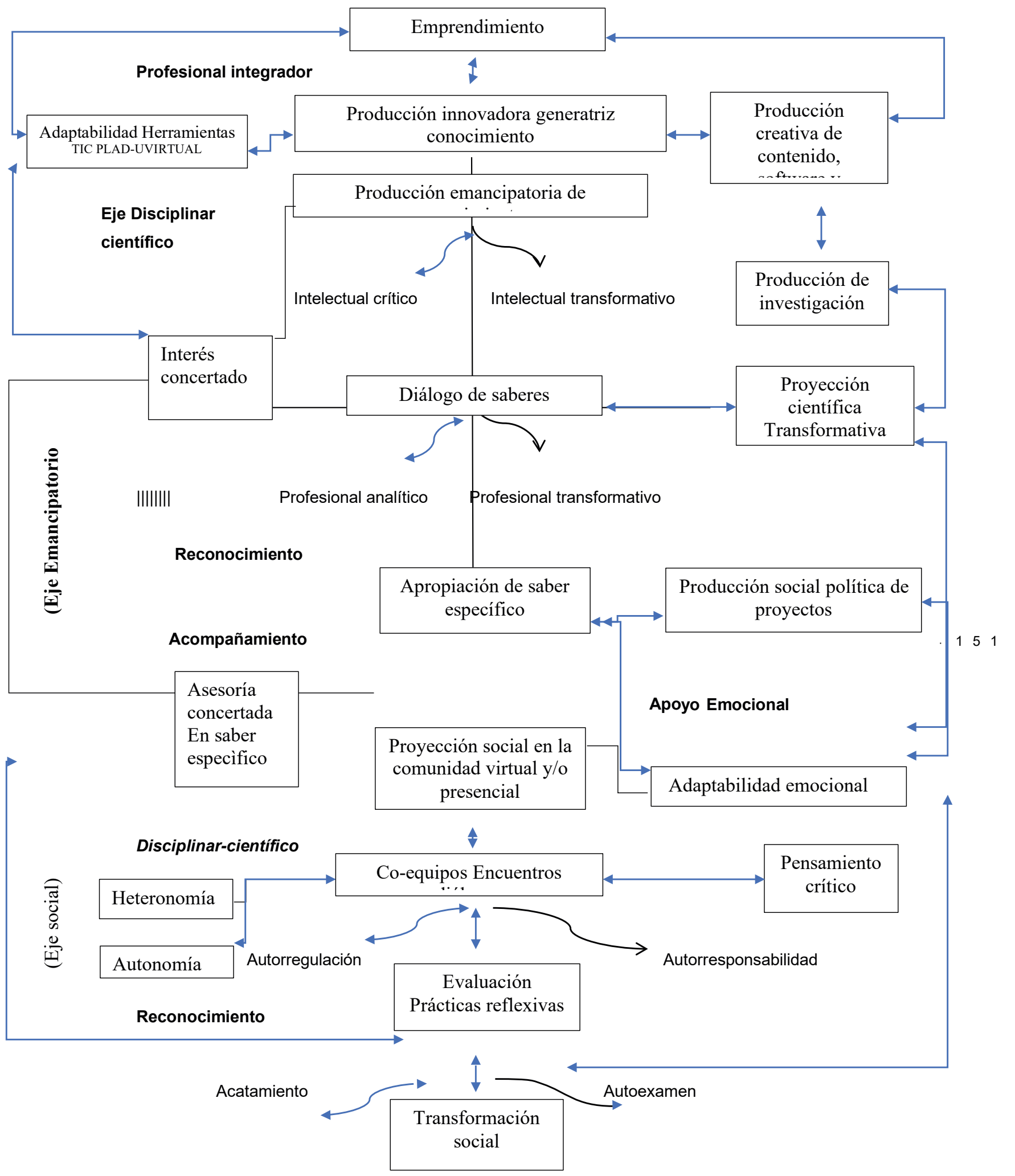

Figura 1. Tendencia curricular para la creación, adaptación y/o uso de tecnologías de la comunicación e información UFPS. Fuente: Adaptado de Saldarriaga (2016) y de Rojas-Suarez y Vergel -Ortega(2019) 
La Socioepistemología [21] fundamenta teóricamente desde un matiz pedagógico en programas académicos con metodologías apoyadas utilizando TIC en la medida que coinciden con su metodología de carácter sistémica, puesto que se focalizan en fenómenos de producción y de difusión del conocimiento desde una vista múltiple, al estudiar la interacción entre epistemología, dimensión sociocultural, procesos cognitivos asociados con desarrollo de competencias específicas y mecanismos de institucionalización vía la enseñanza. A su vez, considera a las prácticas sociales como la base del conocimiento, donde se da importancia toda forma de saber, el cual su fuente puede ser popular, técnica o culta, constituyendo así la sabiduría humana como sustento y la orientación para llevar a cabo una construcción social del conocimiento.

La tendencia anterior, inmersa en un escenario donde el currículo socioepistemológico [21] juega un papel primordial desde las prácticas y la construcción social del conocimiento, buscando respuestas a los problemas del entorno a partir del análisis del contexto real de los actores formadores y en formación, focalizando la atención a mecanismos de institucionalización siempre cuando estén definidos en un currículo considerándolo como una organización social del conocimiento. Cantoral [21], afirma respecto a la concepción de la socioepistemología el recurrir al estudio minucioso de los contextos sociales y culturales vigentes en esos momentos históricos en que se constituyó un saber (historizar), también se exploran otras formas de acercamiento a los fenómenos de construcción del conocimiento (dialectizar), delineando diversidades y posibilidades que la aproximación socioepistemológica provee la ciencia. Esto con el fin de abordar todo tipo de investigación [22] que lleve implícita la construcción de saber [23] en contextos educativos o fuera de ellos [24], en épocas diversas, pasadas o contemporáneas y en escenarios culturales diferenciados.
Enfocada en principios fundamentales interrelacionados: el principio de la racionalidad contextualizada, el principio del relativismo epistemológico, el principio de la resignificación progresiva y el principio normativo de la práctica social. En este sentido, la practica social se convierte en un escenario de aprendizaje para los estudiantes, por ello se presenta como un panorama didáctico para la enseñanza donde el contexto ofrece situaciones que modifican al individuo en colectividad, con la participación de sus pares, compañeros de estudios o demás actores urbanos que fomentan un aprendizaje por adquisición facilitando entre ellos la comunicación de conceptos y procedimientos matemáticos desde consensos que validan un discurso.

La tendencia con eje transversal al enfoque socioepistemológico plantea el desarrollo de habilidades en los estudiantes usando representaciones desde la práctica social y la argumentación científica social, que involucran procesos como medir, predecir, modelar, convenir, asi como transformar. En este horizonte, la argumentación científica como competencia desarrollada en la práctica social reflexiva y crítica de contexto en los estudiantes supone la participación colectiva de acuerdos conceptuales y académicos para la construcción de conocimiento. Desde la óptica de la Socioepistemología confirma las argumentaciones como una construcción sociocultural que responde a la práctica social de la demostración, como mecanismo para validar hipótesis y resultados del conocimiento adquirido por una sociedad. 


\section{CONCLUSIONES}

La tendencia curricular tiene como ejes el eje social, el emancipador, el eje disciplinar y transformador, con objetivos asociados a la producción y diálogo de saberes, producción cientifica, innovadora, investigativa, social polítiva y producción creativa orientada a la transformación social, en escenarios virtuales o de acceso remoto donde el interés concertado prima para lo toma de decisiones, donde la evaluación no la hace un docente sino el equipo que estudia, el grupo de profesionales que apoyan la labor académica e investigativa; la evaluación en escenarios de virtualidad o asincrónicos, va mas allá de un número o una categorización y busca encontrar y potenciar en los seres en formacion, en los futuros profesionales sus pensamientos, sentires, habilidades y capacidades para llevarles a generar nuevo conocimiento a fin de transformar la sociedad que les acoge.

la tendencia incorpora la generación de competencias comunicativas y de difusión de resultados de proyectos de investigación o de procesos de indagación, en una mirada más allá de conocimientos, de contenido sino de gestión del conocimiento, acorde a necesidades y perfiles de formación profesional en el cual toda la comunidad se integra para apoyar perspectivas que potencian y responden a una visión del ciudadano, acciones que gestionan habilidades, conocimientos, de manera que al egresar, pueden comunicar su saber a fin de superar obstáculos epistemológicos presentes en el aprendizaje de las diferentes áreas y ciencias y de aquellos que se encuentran entre el desarrollo tecnológico, el trabajo y el estudio

\section{REFERENCIAS BIBLIOGRÁFICAS}

[1] Coll C (1987), Psicología y Currículum. Una aproximación psicopedagógica a la elaboración del currículum escolar, Paidós, Barcelona. p.31

[2] Gimeno, J. (1988). El curriculum: una reflexión sobre la práctica. Madrid. Morata.

[3] Hernández D, Ferrando M, Soto G, Sainz M and Prieto M 2010 Creatividad, inteligencia sintética y alta habilidad. Reifop 13109

[4] Vergel M, Martínez J and Zafra S (2015) Validez de instrumento para medir la calidad de vida en la juventud: VIHDA Revista Logos Ciencia \& Tecnología 720

[5] Alvis-Puentes, J. F., Aldana-Bermúdez, E., \& Caicedo-Zambrano, S. J. (2019). Los ambientes de aprendizaje reales como estrategia pedagógica para el desarrollo de competencias matemáticas en estudiantes de básica secundaria. Revista de Investigación, Desarrollo e Innovación, 10 (1), 135-147

[6] Rincón O, Vergel M and Zafra S (2017) Modelo de calidad servqual para evaluar factores que influyen en el uso de blogs como recurso didáctico del cálculo. (Bogotá: Editorial Ibáñez)

[7] Zabalza, M. A. (1987): Diseño y desarrollo curricular, Narcea, Madrid.

[8] Balza-Franco, Vladimir; Caro-Ospina, Ana Paola y Navarro-Zúñiga, William (2015) Una mirada a la investigación formativa de pregrado en el área de operaciones y logística de ingeniería industrial en Colombia. Educación en Ingeniería. 10(20):75-87

[9] Watts, M. y Bentley, D. (1991). "Constructivism in the curriculum. Can we close the gap between the strong theoretical version and the weak version of theory-in-practice?" The Curriculum Journal. Vol. 2. Núm. 2. Págs. $171-182$. 
[10] Cabero, J.; Salinas, J.; Duarte, A.; Domingo, J. (2000). Nuevas tecnologías aplicadas a la educación, Madrid, Editorial Síntesis.

[11] Cabero, J.; Martínez, F.; Salinas, J. (2000). Medios audiovisuales y nuevas tecnologías para la formación en el siglo XXI, Murcia, Diego Martín Librero-Editor

[12] Zuluaga Garcés, O. L. (2004). Foucault: una lectura desde la práctica pedagógica. En: Olga Lucía Zuluaga, et al. Pensar de otro modo: usos de Foucault para pensar la educación en Iberoamérica. Seminario Internacional. Bogotá: IDEP. p. 11-37.

[13] Cantón Mayo, I. (2004). "Redes de conocimiento y aprendizaje gestionadas con criterios de calidad". En: Lorenzo Delgado, M.; Ortega Carrillo, J. A.; Sola Martínez, T.; Chacón Medina, A. (coords.). La organización y dirección de redes educativas, Granada, Grupo Editorial Universitario.

[14] Salazar J and Vergel M (2018) Hermeneusis de la práctica pedagógica y formación de estudiantes matemáticamente competentes. En Investigación y praxis en la enseñanza de las matemáticas. (Cúcuta: Universidad Simón Bolívar).

[15] Saldarriaga, O. (2008). Cuadernos de psicopedagogía. 5 .

[16] Parra, H., Suarez, J., y Vergel M. Curricular trends in the Universidad Francisco de Paula Santander academic program offerings 2019 Journal of Physics Conference Series 1329:012013 DOI: 10.1088/1742$\underline{6596 / 1329 / 1 / 012013}$

[17] Martínez, J., Vergel, M. \& Zafra, S. (2015). Validez de instrumento para medir la calidad de vida en la juventud: VIHDA. Revista Logos Ciencia \& Tecnología, 7(1), 20-28.
[18] Martínez, J., Zafra, S. \& Vergel, M. (2016). Comportamiento juvenil y competencias pro sociales. Bogotá: Ibáñez.

[19] Martínez Lozano, J., Vergel, M. \& Zafra Tristancho, S. (2016). Factores asociados al rendimiento académico en adultos. Revista Científica, 2(25). doi: http://dx.doi.org/10.14483// udistrital.jour.RC.2016. 25.a4

[20] Vergel, M., Rojas J.P. y Urbina J. (2020). Imaginario de universidad en jovenes sordos. Bogotá: ECOE Ediciones.

[21] Paz-Corrales, Luis; Cantoral, Ricardo (2019). Estudio socioepistemológico sobre la confrontación entre la geometría de descartes y la geometría analítica. En Pérez-Vera, Iván Esteban; García, Daysi (Eds.), Acta Latinoamericana de Matemática Educativa (pp. 394-403). México, DF: Comité Latinoamericano de Matemática Educativa

[22] Martínez J, Vergel $M$ and Zafra $S$ (2015) Comportamiento juvenil y competencias prosociales (Bogotá: Editorial Ibáñez)

[23] Pilonieta, L., Martinez J., y Vergel Ortega, M. (2020). Ineamientos de enseñanza a estudaintes con hipoacusia. Bogotá: ECOE ediciones.

[24] Vergel M, Martínez J and Zafra S (2015) Apps en el rendimiento académico y auto concepto de estudiantes de ingeniería Revista Logos Ciencia \& Tecnología 6198 